\title{
Comparison of single-step reverse transepithelial all-surface laser ablation (ASLA) to alcohol- assisted photorefractive keratectomy
}

This article was published in the following Dove Press journal:

Clinical Ophthalmology

26 June 2012

Number of times this article has been viewed

\author{
loannis M Aslanides' \\ Sara Padroni' \\ Samuel Arba Mosquera ${ }^{2}$ \\ Antonis loannides' \\ Achyut Mukherjee' \\ 'Emmetropia Mediterranean Eye \\ Institute, Heraklion, Crete, Greece; \\ ${ }^{2}$ Schwind eye-tech-solutions $\mathrm{GmbH}$, \\ Kleinostheim, Germany
}

Purpose: To evaluate postoperative pain, corneal epithelial healing, development of corneal haze, refractive outcomes, and corneal aberrations in a novel one-step, modified transepithelial photorefractive keratectomy (PRK), termed All-surface laser ablation (ASLA), compared to conventional, alcohol-assisted PRK.

Materials and methods: Sixty eyes of 30 myopic patients were prospectively recruited to a randomized fellow eye study. Patients underwent conventional alcohol-assisted PRK in one eye (control group) and ASLA-modified transepithelial PRK in the other (30 eyes in each treatment arm). Primary endpoints were postoperative pain and haze scores at 1 day, 3 days, 1 week, and 1, 3, 6, and 12 months. Secondary endpoints included visual acuity at 1, 3, 6, and 12 months, corneal aberrations at 3, 6, and 12 months, and early and late onset haze. Refractive predictability, safety, and efficacy of the two methods were considered.

Results: The average age of the cohort was 29 years (standard deviation [SD]: 9; range: 18-46), and the average spherical equivalent refractive error was -4.18 diopters (SD: 1.9). At 3 days after surgery, the average pain score was $64 \%$ lower in the ASLA group $(P<0.0005)$. At this point, $96 \%$ of ASLA eyes had no epithelial defect, whereas $43 \%$ in the alcohol-assisted group did not achieve complete epithelial healing, and required replacement of bandage contact lens. The haze level was consistently lower in the ASLA group at all time points from 1 to 6 months.

Conclusion: This study shows that the ASLA technique may have a future role in refractive surgery, due to the fact that it offers faster epithelial healing, lower pain scores, and significantly less haze formation.

Keywords: cornea, ASLA, PRK, alcohol, transepithelial, epithelium, laser

\section{Introduction}

Photorefractive keratectomy (PRK) was introduced more than 25 years ago as a corneal refractive surgical technique using the excimer laser. ${ }^{1,2}$ The advent of laser-assisted in situ keratomileusis (LASIK) led to a decline in the popularity of PRK. ${ }^{3}$ Nevertheless, there is a recognition that surface ablation has several potential advantages with regard to preserving corneal biomechanical integrity and avoiding intraoperative and late flap-related complications. There has thus been a resurgence in surface ablation techniques. The main limitations of PRK remain - postoperative pain, delayed epithelial healing, and postoperative stromal haze. Several modifications of the technique have evolved to partly address these shortcomings. A key area is the technique of epithelial removal. The use of alcohol as an alternative to mechanical debridement has been assessed, as has preservation of the epithelium as a flap (laser-assisted sub-epithelial keratectomy [LASEK] and Epi-LASIK). ${ }^{4}$ Another alternative is transepithelial PRK,
Correspondence: loannis M Aslanides Emmetropia Mediterranean Eye Institute, Elephteria Square, 44 Heraklion,

Crete 7|20I, Greece

Tel +302810226198

$\mathrm{Fax}+302810343436$

Email i.aslanides@emmetropia.gr 
where epithelial removal is generally carried out with laser phototherapeutic (PTK) ablation, followed by a stromal laser refractive ablation. ${ }^{5-8}$ Transepithelial PRK has several advantages, including no instrument contact with the eye, reduced intervention time, and the potential to minimize the size of an epithelial defect to that required for stromal ablation, as well as the avoidance of alcohol and, thus, potential toxicity. A number of previous studies have assessed such a two-step approach - an initial PTK pre-treatment for epithelial removal, followed by PRK. ${ }^{5,7}$ Other studies have examined transepithelial PRK (as a two-step procedure) versus alcohol-assisted PRK. ${ }^{8-10}$

In this study, we evaluated a new technique for combined epithelial and stromal laser ablation. This has been termed All-surface laser ablation (ASLA), a modified transepithelial PRK technique. The technique differs from the previously described transepithelial PRK technique in a number of aspects. Firstly, the epithelium is not ablated using an even or broad beam PTK profile. Instead a custom epithelial profile has been generated from population studies, which have demonstrated that the epithelium does not have a uniform thickness. Studies using high frequency ultrasound have been utilized to form a standard epithelial model, demonstrating relatively thicker peripheral epithelium. ${ }^{11}$ The ablation profile generated from this population model thus targets $55 \mu \mathrm{m}$ centrally, and $65 \mu \mathrm{m}$ peripherally, for an $8 \mathrm{~mm}$ ablation zone, with further consideration to the differential ablation rate in epithelium, compared to stroma. Secondly, the ablation is not carried out as two distinct steps, but rather as a single continuous profile, mitigating the risk of dehydration between steps. Thirdly, the profile uniquely applies the stromal component of the ablation first, over the epithelium, prior to the additive epithelial profile. This has the potential benefit of maximising the smoothing effect of the epithelium, since it is recognized that epithelial thickening occurs in areas of stromal irregularity. This potential advantage is negated when the epithelium is removed prior to stromal ablation, thus exposing the underlying irregularity.

In addition to the use of this novel surface ablation technique, we also used adjunctive mitomycin $\mathrm{C}$ (MMC). This is recognized as a means for reducing the occurrence of postoperative stromal haze, thereby addressing the other main limitation of surface ablation techniques. ${ }^{12}$

The primary aim of this study was to assess postoperative pain, photophobia, and stromal haze, compared to a control group undergoing standard alcohol-assisted epithelial removal. Additionally, we aimed to assess visual and refractive outcomes, and safety, compared to the controls. We also sought to evaluate the relative effects of ASLA transepithelial ablation when MMC is used.

\section{Materials and methods}

The patients of this study were recruited in Emmetropia Mediterranean Eye Institute, a private refractive practice in Crete, from September 2010 to March 2011. Written informed consent was obtained prior to inclusion, and the study was performed following the mandates of the Helsinki Agreement. Prior ethical approval was obtained from the local Clinical Ethics Committee.

The study was designed as a prospective interventional study with contralateral eye control. The choice of study design was based on the need for a matched control group, given the small numbers. In addition, there was the advantage of having the same patient to subjectively compare and evaluate the treatment modalities between eyes. For each patient, the choice of first eye treated and the application of intervention or control was randomized using randomization tables, with the fellow eye receiving the alternate treatment. Patients and observers were masked for all features other than slit-lamp examination, which could not be masked due to differing clinical appearances.

Inclusion criteria were: age over 18; myopic refraction with astigmatism no higher than 3 diopters (D); stable refraction for 12 months preceding surgery; maximum planned corneal stromal ablation of $110 \mu \mathrm{m}$; and corrected Snellen distance visual acuity (CDVA) of 0.7 Snellen fraction or better. Eyes with corneal epithelial pathology, keratoconus, ocular inflammation, glaucoma, and posterior segment pathology were excluded from the study. All patients received bilateral treatment by the same surgeon at the same session.

The preoperative assessment included uncorrected distance acuity (UDVA) and CDVA, manifest and cycloplegic refraction, ultrasound pachymetry, corneal topography with Orbscan ${ }^{\circledR}$ (Bausch and Lomb, Rochester, NY) and Keratron Scout Topographer (Optikon SpA, Rome, Italy). Corneal aberrations were measured with the Keratron Scout, and ocular aberration measurements with the Ork Wavefront Analyzer (SCHWIND eye-tech-solutions GmbH, Kleinostheim, Germany). Both were analyzed at $6 \mathrm{~mm}$ diameter. Slit lamp exam, including dilated fundus assessment, was performed. The total surgical time from speculum insertion to removal was recorded in the operating theatre with a stopwatch.

In both groups, surgical preparation was with iodine and topical tetracaine $0.5 \%$ instillation, prior to draping 
and insertion of a speculum. In the alcohol group, epithelial delamination was performed with $20 \%$ ethanol, applied using an $8 \mathrm{~mm}$ well, followed by epithelial removal with a spatula. ${ }^{8}$ In the ASLA group, treatment was preceded by standardized wet sponge application: a Merocel ${ }^{\circledR}$ sponge (Medtronic, Minneapolis, MN) was dipped in balanced salt solution and left to expand maximally, then applied with three slow "painting" movements on the corneal surface. This step avoids uneven wetting, and the subsequent risk of uneven ablation. All patients underwent treatment with the Amaris $500 \mathrm{~Hz}$ excimer laser (SCHWIND eye-tech-solutions). In the PRK group, treatment was with standard PRK protocol. In the ASLA group, the excimer laser was administered in a single continuous session, as detailed above, with combined epithelial and stromal profile. In both groups, ablation profiles were aspheric aberration neutral (ie, nonwavefront-guided treatment).

The postoperative regime was consistent between groups. Before applying a contact lens (Frequency ${ }^{\circledR}$ Aspheric; Coopervision, Pleasanton, CA), 1 drop of topical ketorolac $0.5 \%$, and 1 drop of ofloxacin $0.3 \%$ were applied. After surgery, the patients were treated with topical instillation of ofloxacin $0.3 \%$ four times daily (until removal of the contact lens), dexamethasone $0.1 \%$ drops four times daily (reducing over 12 weeks), and artificial tear drops four times daily for 3 months.

For all eyes in both groups, where the stromal ablation was planned to be more than $75 \mu \mathrm{m}$, the patient received a standardized intraoperative application of $0.02 \% \mathrm{MMC}$, applied for 30 seconds using an MMC-impregnated sponge pledget, immediately after excimer ablation, followed by copious saline irrigation. ${ }^{12}$ Twenty-eight eyes of 14 patients (46\%), in both groups, received bilateral MMC after laser ablation. Postoperative assessment was performed on day 1 , day 3, at 1 week, and 1, 3, 6, and 12 months after surgery. Patients were masked to the assignment of eyes, while the treating and observing clinicians could not be. At each postoperative visit, UDVA and CDVA were recorded. During the first three follow-up visits, subjective pain and photophobia were assessed by patients scoring each eye on a scale of $0-10$. Postoperative corneal haze at 1 week, and 1, 3, 6, and 12 months was graded at the slit lamp microscope, according to the Fantes scale, by two independent ophthalmologists, and the average of the two values taken. ${ }^{13}$ Contact lens replacement and early re-epithelisation were noted. Subjective refraction and corneal aberrations were recorded preoperatively and at 1, 3, 6, and 12 months postoperatively.
All statistical analyses were performed using Microsoft ${ }^{\circledR}$ Excel (Microsoft Corporation, Redmond, WA). Intragroup variables were compared using unpaired $t$-tests, while temporal changes were compared using paired $t$-tests. A $P$ value less than 0.05 was considered statistically significant.

\section{Results}

\section{Baseline demographics and control group equivalence}

Sixty eyes of 30 myopic patients (19 male and 11 female) undergoing excimer photorefractive keratectomy were included. The average age was 29 years (SD: 9; range: 17-46). Preoperative mean UDVA was 0.01 Snellen fraction (SD: 0.02 ; range: $0-0.1$ ), in the PRK group, and 0.02 (SD: 0.11 ; range: $0-0.6)$ in the ASLA group $(P=0.2)$. Preoperative mean CDVA was 0.98 Snellen fraction (SD: 0.06; range: $0.7-1.0$ ) in the PRK group, and 0.96 (SD: 0.08 ; range: $0.7-1)$ in the ASLA group $(P=0.2)$. Mean treated refractive error was $-4.09 \mathrm{D}$ (SD: 1.87$)$ in the PRK group, and $-4.25 \mathrm{D}$ (SD: 1.9) in the ASLA group, with a mean difference between pairs of intervention and control eyes of $0.65 \mathrm{D}$ (SD: 0.63). Preoperative corneal higher order aberrations, including spherical aberration, coma, and trefoil did not differ between the PRK and ASLA groups ( $P=0.2$ for all variables) (Table 1). The average stromal ablation was $72.77 \mu \mathrm{m}$ (SD: 28.4) in the PRK group and $71.33 \mu \mathrm{m}$ (SD 26.3) in the ASLA group $(P=0.4)$. Both eyes received essentially identical optical zone (OZ), transition zone (TZ), and total ablation zone (TAZ). (OZ: $6.36 \pm 0.28 \mathrm{~mm}$ versus $6.35 \pm 0.27 \mathrm{~mm}$; TZ: $1.28 \pm 0.48 \mathrm{~mm}$ versus $1.24 \pm 0.46 \mathrm{~mm}$; TAZ: $7.64 \pm 0.48 \mathrm{~mm}$ versus $7.59 \pm 0.43 \mathrm{~mm}$ for PRK and ASLA, respectively) ( $P=0.4$ for all variables). Fourteen (47\%) patients, with PRK and ASLA in fellow eyes, received MMC bilaterally, immediately after ablation; the rest did not

Table I Higher order aberrations in single-step PRK and alcoholassisted PRK

\begin{tabular}{|c|c|c|c|c|c|c|}
\hline & \multicolumn{2}{|c|}{ Spherical } & \multicolumn{2}{|l|}{ Coma } & \multicolumn{2}{|c|}{ Trefoil } \\
\hline & PRK & ASLA & PRK & ASLA & PRK & ASLA \\
\hline \multirow[t]{2}{*}{ Preoperatively } & 0.49 & 0.57 & 0.27 & 0.33 & 0.22 & 0.20 \\
\hline & $(0.21)$ & $(0.35)$ & $(0.14)$ & $(0.17)$ & $(0.13)$ & $(0.12)$ \\
\hline \multirow[t]{2}{*}{ At I month } & 0.85 & 0.80 & 0.32 & 0.32 & 0.25 & 0.21 \\
\hline & $(0.65)$ & $(0.63)$ & $(0.22)$ & $(0.18)$ & $(0.18)$ & $(0.21)$ \\
\hline \multirow[t]{2}{*}{ At 6 months } & 0.73 & 0.68 & 0.33 & 0.32 & 0.20 & 0.17 \\
\hline & $(0.36)$ & $(0.33)$ & $(0.24)$ & $(0.17)$ & $(0.13)$ & $(0.13)$ \\
\hline \multirow[t]{2}{*}{ At I year } & 0.77 & 0.73 & 0.43 & 0.27 & 0.18 & 0.23 \\
\hline & $(0.30)$ & $(0.34)$ & $(0.22)$ & $(0.18)$ & $(0.1 \mathrm{I})$ & $(0.19)$ \\
\hline
\end{tabular}

Note: Standard deviations are shown in parentheses.

Abbreviations: PRK, transepithelial photorefractive keratectomy; ASLA, all-surface laser ablation. 
receive $\mathrm{MMC}$ in either eye. A small subset of patients $(\mathrm{N}=5)$ had operative times recorded. Approximate surgery time for the ASLA patients was 1 minute, compared to 3 minutes for PRK. There were no intraoperative complications. No patients were lost to follow-up.

\section{Postoperative pain}

At day 3, pain scores were statistically significantly lower in the ASLA group, at 0.9 (SD: 1.6; range: 0-8), compared to 2.6 (SD: 1.7; range: $0-6)$ in the PRK group $(P<0.0005)$. There was no significant difference in the very early pain scores at the first postoperative day: 4.5 (SD: 2.6; range: $0-10)$ in the ASLA group $(P=0.3)$, and 4.1 (SD: 2.6; range: $0-8)$ in the PRK group (Figure 1B).

\section{Postoperative photophobia}

Day 1 photophobia scores were similar between groups at 6.2 (SD: 2.4) in the PRK group, and 6.1 (SD: 2.6) in the ASLA group $(P=0.4)$. At the third postoperative day, the difference remained negligible: 1.8 (SD: 2.5 ) in the ASLA group, compared to 2.2 (SD: 2.1$)$ in the PRK group $(P=0.6)$.

\section{Epithelial healing}

By day 3, significantly more eyes in the ASLA group had epithelial closure (97\%) compared to the alcohol group, where only 17 eyes (57\%) had a healed epithelium $(P=0.0002$ [Chi-square test]). This was also reflected in the need for a further bandage contact lens at this time; $43 \%$ of eyes treated with conventional (alcohol-assisted) PRK, but only $3 \%$ in the ASLA group required replacement of contact lens. At 1 week, all eyes in both groups had epithelial closure. There was no clear effect of MMC on epithelial healing, with 19 of $28(68 \%)$ eyes exposed to MMC epithelialising at day 3 versus $27 / 32$ (84\%) in untreated eyes $(P=0.13)$.

\section{Corneal haze}

At 1 week, haze scores averaged 0.3 (SD: 0.6; range: $0-3$ ) in the PRK group and 0.1 (SD: 0.3; range: $0-1)$ in the ASLA group $(P=0.07)$. By 1 month, this difference was statistically significant, with a mean score of 0.4 (SD: 0.8 ; range $0-3$ ) in the PRK group, and 0.2 (SD: 0.5; range: $0-2$ ) in the ASLA group $(P<0.05)$. Significantly reduced haze in the ASLA group persisted at 3 and 6 months $(P<0.05)$, although by 1 year, all eyes in either group had a score of 0 . There was no evidence of increased corneal haze in eyes with greater ablation depths with our protocol, where all ablations $>75 \mu \mathrm{m}$ received MMC treatment $(P>0.05)$ (Figure 1A).
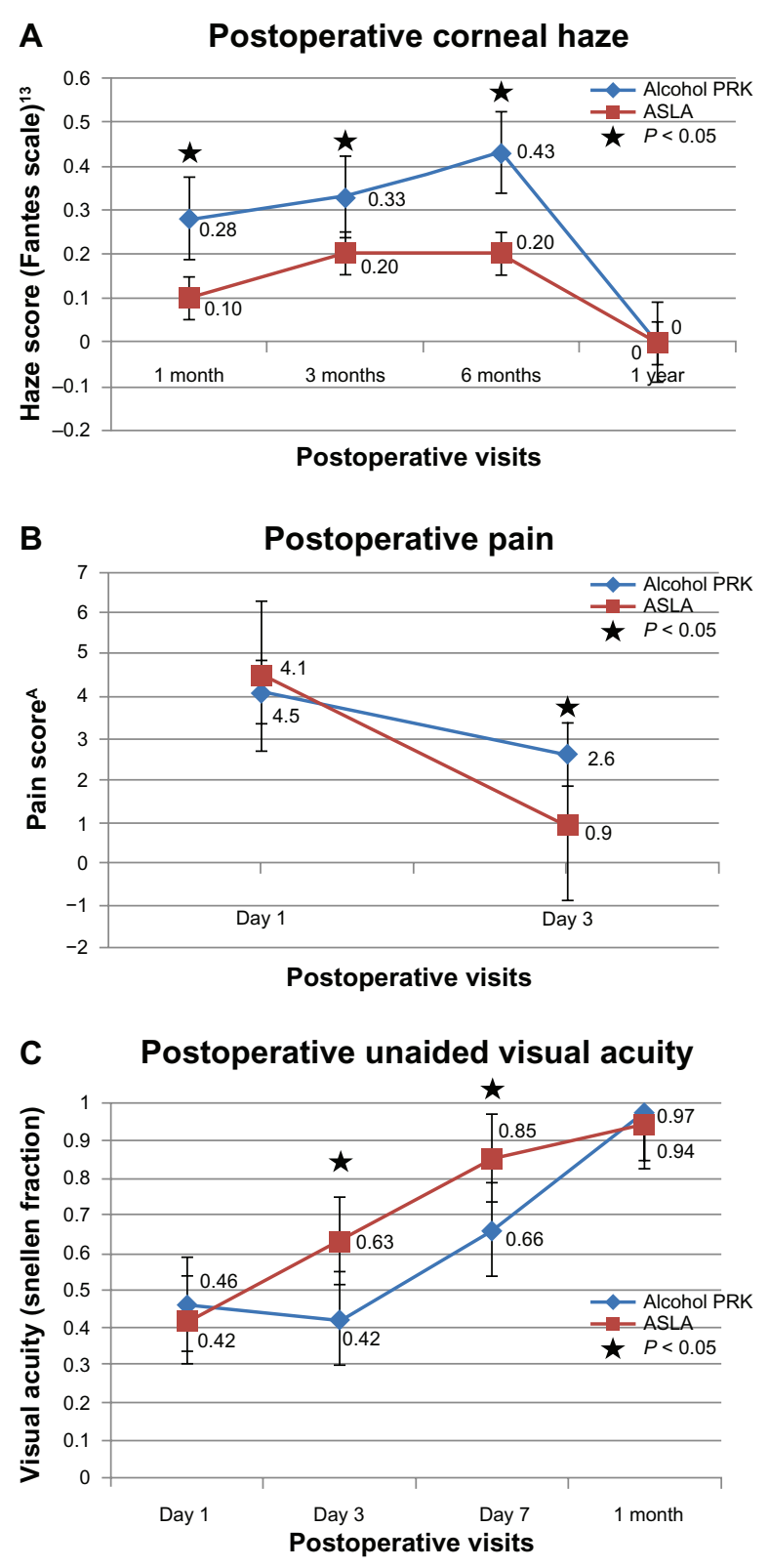

Figure I Postoperative outcomes in alcohol-assisted PRK versus ASLA.

Notes: ASubjective analogue scale I to 10 . There is significantly reduced corneal haze at I, 3, and 6 months, reduced day 3 postoperative pain, and improved unaided visual acuity in the PRK group.

Abbreviations: PRK, transepithelial photorefractive keratectomy; ASLA, allsurface laser ablation.

\section{Uncorrected vision and refraction}

Day 1 mean UDVA was similar at 0.42 (SD: 0.18; range: $0.1-0.7$ ) in the ASLA group, and 0.46 (SD: 0.19; range: $0.1-0.9)$ in the PRK group $(P=0.2)$. However, by day 3, mean UDVA was better in the ASLA group at 0.63 (SD: 0.15; range: $0.3-0.9$ ) versus 0.42 (SD: 0.15 ; range: $0.2-0.8$ ) in the PRK group $(P<0.05)$. At this third postoperative day, $90 \%$ (27) of eyes undergoing ASLA achieved 0.7 or better vision, compared to $43 \%$ of PRK treated eyes. This statistically significant early visual recovery in the ASLA group persisted at 
1 week, with mean UDVA of 0.85 (SD: 0.19; range: 1.2-0.4), compared to 0.66 (SD: 0.24; range 1.0-0.1) in the PRK group $(P<0.0001)$. At 1 month, mean UDVA was 0.94 (SD: 0.1; range: 1.0-0.6) in the PRK group, and 0.97 (SD: 0.1; range: $1.2-0.6)$ in the ASLA group, with no remaining difference $(P=0.1)$. There remained no difference at 3, 6, and 12 months ( $P=0.5$ at all time points) (Figure 1C). The levels of accuracy for both techniques were high, with no statistically significant difference in attempted versus achieved final refraction (Figures 2 and 3). Both techniques were equivalent in safety, with one eye in each group losing one line.

\section{Higher order aberrations}

There were no differences between groups noted with regard to higher order aberrations, measured at any time point. There was a significant increase $(P<0.05)$ in spherical higher order aberration postoperatively, equivalent in both groups (Table 1).

\section{Discussion}

In this study, we sought to assess a modified transepithelial PRK technique, with a customized epithelial ablation profile (ASLA), with respect to the main current limitations of surface ablation techniques, namely postoperative pain/ photophobia, haze, and delayed visual recovery., ${ }^{4,7,12,14,15}$
The primary finding of this study was that the use of this technique offers patients significantly reduced early postoperative pain (with a mean main score of 0.9 versus 2.6 at the third postoperative day), reduced photophobia, and more rapid epithelial closure, compared to conventional alcohol-assisted PRK. A specific practical resultant benefit was that ASLA required bandage contact lens replacement in only one eye (3\%) by day 3 , whereas, in the PRK group, the lens had to be replaced in $43 \%$ of eyes. With regard to early visual recovery, there is also a significant improvement, with approximately three Snellen lines better vision on Day 3 in the ASLA group $(0.4$ versus $0.2 ; P<0.05)$. It was one month before the PRK group approached the ASLA group, in terms of vision $(P=0.08)$.

This improvement on PRK's limitations occurs at no cost to outcomes: the ASLA technique was equivalent or superior to the conventional alcohol-assisted PRK in all metrics. With specific reference to safety, there was no difference, whereas the ASLA group appeared to have better outcomes, with $33 \%$ versus $13 \%$ having improved CDVA $(P>0.05)$. There was no observable difference in induced higher order aberrations between groups.

The main further limitation of conventional PRK is postoperative haze. ${ }^{12,14,15} \mathrm{~A}$ key finding of this study is that the use of ASLA transepithelial treatment seems to offer

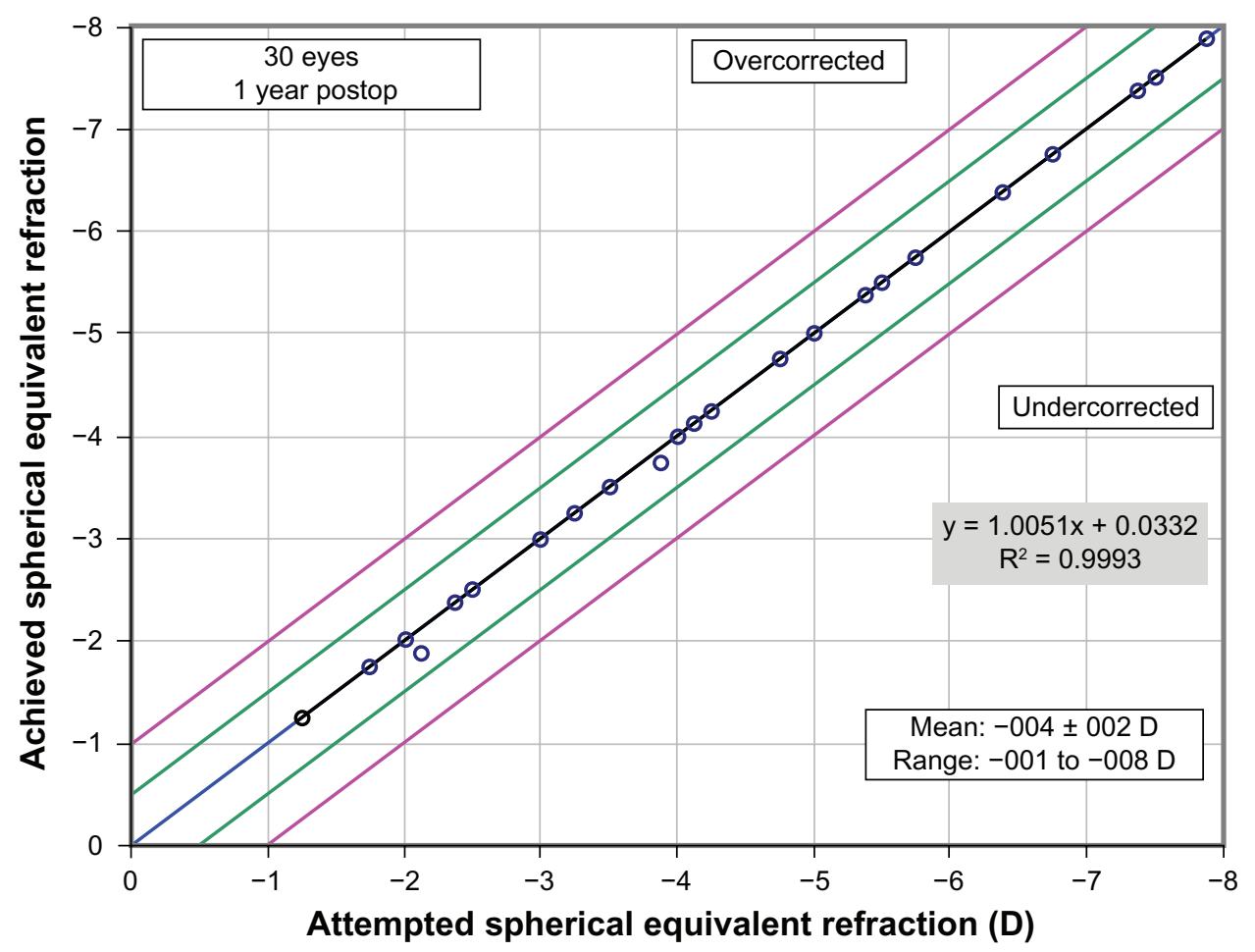

Figure 2 Attempted versus achieved spherical equivalent refraction: PRK/ASLA.

Abbreviations: PRK, transepithelial photorefractive keratectomy; ASLA, all-surface laser ablation; D, diopters. 


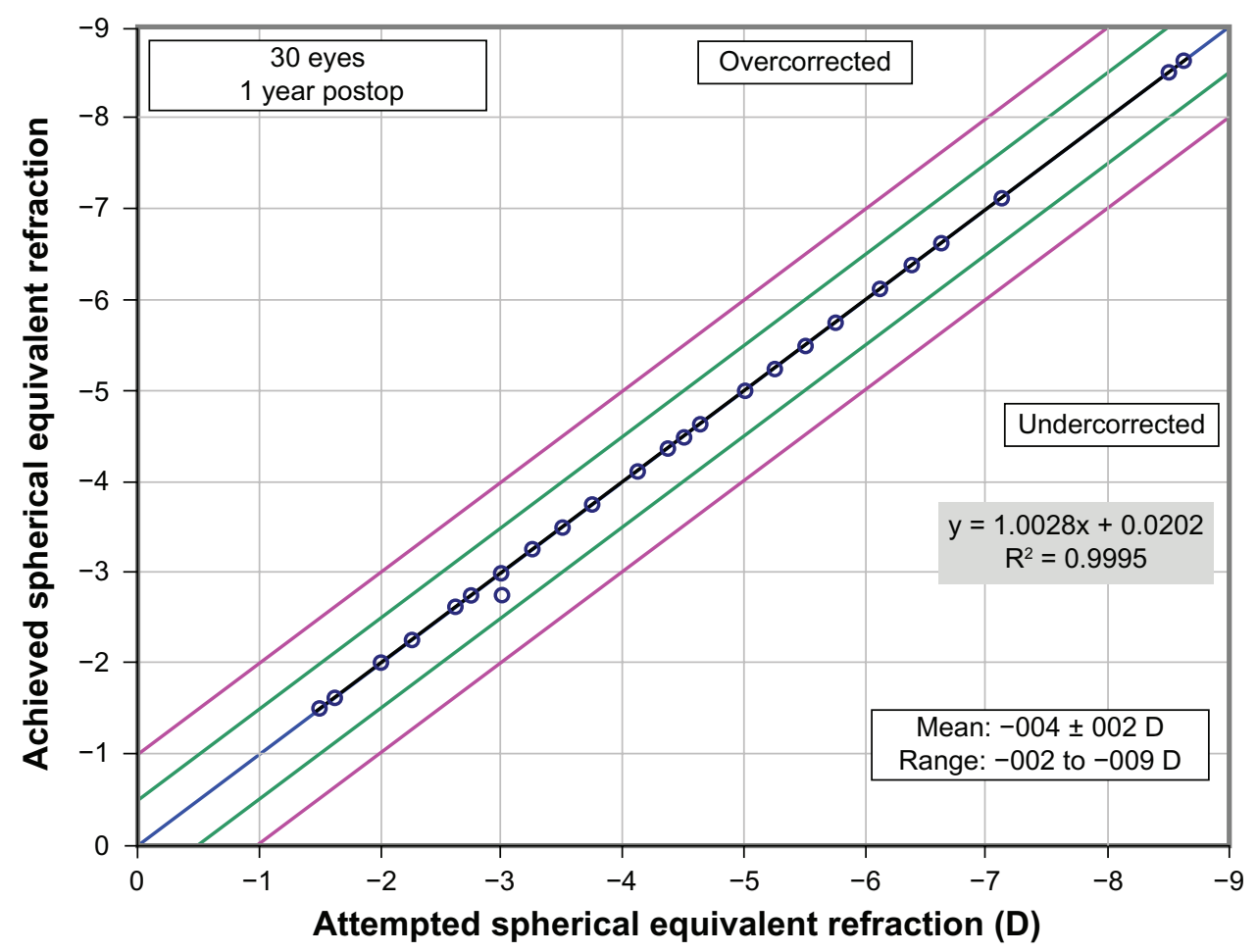

Figure 3 Attempted versus achieved spherical equivalent refraction: alcohol-assisted PRK. Abbreviations: PRK, photorefractive keratectomy; D, diopters.

some further additional benefit over and above that provided by MMC, which was used equivalently in both groups. Haze is a relatively rare side effect of PRK, associated with high ablations, discontinuation of steroids, postoperative UV exposure, and male sex. ${ }^{14,15} \mathrm{~A}$ key turning point in the evolution of surface ablation treatments has been the introduction of MMC adjunctive therapy, which has gone some way towards ameliorating postoperative haze, and has been pivotal to the current resurgence of PRK. The outcomes of MMC use in refractive transepithelial PRK have not been previously reported. Notably, objective postoperative haze was less in the ASLA group at 1 week $(P=0.07)$, and 1,3 , and 6 months $(P<0.05)$, although absent in both groups at 1 year. In previous studies, the peak of corneal haze was observed at a mean time of 7.4 months. ${ }^{15}$ The ASLA cohort had consistently lower levels of haze over a 6-month period, which reached a plateau after the third month, with no attributable visual deficit.

Existing literature reports have varying findings with regard to transepithelial PRK. It has previously been shown to be effective in the management of keratoplasty and postrefractive surgery complex errors. ${ }^{5,6} \mathrm{~A}$ small study comparing alcohol-assisted versus PTK epithelial removal found no difference in healing time, and greater pain in the transepithelial treatment. ${ }^{8}$ Another comparison of transepithelial PRK, mechanical removal PRK, and LASEK found no difference in pain or haze. ${ }^{10} \mathrm{~A}$ large retrospective comparison of transpithelial PRK with epi-LASIK, LASEK, and LASIK found better visual outcomes with transepithelial treatment for high myopia. ${ }^{9}$

These previous studies, however, utilized the two-step technique, of PTK followed by PRK. ${ }^{5,6,8,10,16}$ This is distinct from our reported ASLA transepithelial PRK, which uses a population-based epithelium-removal profile, rather than PTK, and a single-step ablation. Moreover, during ASLA, first the refractive component, and then the epithelial profile is ablated. So, the refractive ablation is applied over and completely or incompletely removes epithelium, whereas the subsequent epithelial profile advances the curvature change down to stroma. This reverse sequence maximizes the smoothing effect of epithelium over stromal irregularities, and utilizes the early period of expected better cooperation and fixation for the critical refractive ablation, leaving the less alignment-critical profile for the end. Another advantage is the rapid treatment time; we found that the one-step laser treatment takes considerably less time than either alcohol removal or two-step PTK laser removal. This prevents issues with dehydration prior to ablation. A single previous study has assessed the ASLA technique. However, the follow-up period was limited to 3 months, and the use of a retrospective control 
group introduces potential bias during patient selection and consent, which are avoided in our fellow eye methodology. ${ }^{17}$ A key difference in the findings was early visual recovery, which was superior in the alcohol group in the above study, compared to ours. However, unlike our study, MMC was not used.

It is known that keratocyte apoptosis and myofibroblast activation is a key factor in stromal recovery after surface ablation procedures, and that transepithelial ablation results in lower levels of keratocyte apoptosis. ${ }^{18,19}$ Epithelial regeneration and integrity is paramount, in order to initiate cytokine induction, which in turn activates stromal wound healing. ${ }^{20}$ Animal studies confirm that transepithelial epithelium removal produces a smooth uniform surface, ideal for epithelial regeneration. ${ }^{21}$ Although alcohol delamination of the corneal epithelium can provide a smooth surface at the level of the Bowman's membrane, the viability of the epithelial cells is reduced, with potential limbal effects. ${ }^{20,22}$ Agrawal et al observed an increased inflammatory response, as well as damage to the anterior stromal keratocytes, after alcohol exposure, which could potentiate haze formation. ${ }^{23}$ We believe that the lower scores for both early- and late-onset corneal haze in ASLA-treated eyes are due to a smoother stromal bed, facilitating centripetal growth of new epithelium, faster treatment time, and optimal epithelial healing.

The main limitation of this study relates to sample size, although, even in this relatively small sample, the study was sufficiently powered to demonstrate a statistically significant improvement in early recovery with ASLA transepithelial PRK.

\section{Conclusion}

All-surface laser ablation, a no-touch, single-step transepithelial PRK technique, demonstrates significantly faster epithelial healing, reduced corneal haze, and reduced postoperative pain, compared with alcohol-assisted PRK. The refractive and visual outcomes are at least equivalent to those of alcohol-assisted PRK. Additional benefits are reduced patient contact and faster operating time. Further scientific evaluation is warranted.

\section{Disclosure}

Samuel Arba Mosquera is an employee of SCHWIND eyetech-solutions $\mathrm{GmbH}$. The authors report no other conflicts of interest in this work.

\section{References}

1. Marshall J, Trokel S, Rothery S, Krueger R. Photoablative reprofiling of the cornea using an excimer laser: photorefractive keratectomy. Lasers Ophthalmol. 1986;1:21-48.
2. Trokel SL, Srinivasan R, Braren B. Excimer laser surgery of the cornea. Am J Ophthalmol. 1983;96(6):710-715.

3. Duffey RJ, Leaming D. US trends in refractive surgery: 2004 ISRS/ AAO Survey. J Refract Surg. 1995;21(6):742-748.

4. Ghoreishi M, Attarzadeh H. Alcohol-assisted versus mechanical epithelium removal in photorefractive keratectomy. J Ophthalmic Vis Res. 2010;5(4):223-227.

5. Pedrotti E, Sbabo A, Marchini G. Customized transepithelial photorefractive keratectomy for iatrogenic ametropia after penetrating or deep lamellar keratoplasty. J Cataract Refract Surg. 2006;32:1288-1291.

6. Camellin M, Arba Mosquera S. Simultaneous aspheric wavefrontguided transepithelial photorefractive keratectomy and phototherapeutic keratectomy to correct aberrations and refractive errors after corneal surgery. J Cataract Refract Surg. 2010;36(7):1173-1180.

7. La Tegola MG, Alessio G, Sborgia C. Topographic customized photorefractive keratectomy for regular and irregular astigmatism after penetrating keratoplasty using the LIGI CIPTA/LaserSight platform. J Refract Surg. 2007;23(7):681-693.

8. Kanitkar KD, Camp J, Humble H, Shen DJ, Wang MX. Pain after epithelial removal by ethanol-assisted mechanical versus transepithelial excimer laser debridement. J Refract Surg. 2000;16(5):519-522.

9. Ghadhfan F, Al-Rajhi A, Wagoner MD. Laser in situ keratomileusis versus surface ablation: visual outcomes and complications. J Cataract Refract Surg. 2007;33(12):2041-2048.

10. Lee HK, Lee KS, Kim JK, et al. Epithelial healing and clinical outcomes in excimer laser photorefractive surgery following three epithelial removal techniques: mechanical, alcohol, and excimer laser. $\mathrm{Am} \mathrm{J}$ Ophthalmol. 2005;139(1):56-63.

11. Reinstein DZ, Archer TJ, Gobbe M, Silverman RH, Coleman DJ. Epithelial thickness in the normal cornea: three-dimensional display with Artemis very high-frequency digital ultrasound. J Refract Surg. 2008;24(6):571-581.

12. Carones F, Vigo L, Scandola E, Vacchini L. Evaluation of the prophylactic use of mitomycin-C to inhibit haze formation after photorefractive keratectomy. J Cataract Refract Surg. 2002;28(12):2088-2095.

13. Fantes FE, Hanna KD, Waring GO, et al. Wound healing after excimer laser keratomileusis (photorefractive keratectomy) in monkeys. Arch Ophthalmol. 1990;108(5):665-675.

14. Caubet E. Course of subepithelial corneal haze over 18 months after photorefractive keratectomy for myopia [corrected]. Refract Corneal Surg.1993;9(Suppl 2):S65-S70.

15. Kuo IC, Lee SM, Hwang DG. Late-onset corneal haze and myopic regression after photorefractive keratectomy (PRK). Cornea. 2004;23(4):350-355.

16. Buzzonetti L, Petrocelli G, Laborante A, et al. A new transepithelial phototherapeutic keratectomy mode using the NIDEK CXIII excimer laser. J Refract Surg. 2009;25(Suppl 1):S122-S124.

17. Fadlallah A, Fahed D, Khalil K, et al. Transepithelial photorefractive keratectomy: clinical results. J Cataract Refract Surg. 2011;37(10):1852-1857.

18. Helena MC, Baerveldt F, Kim WJ, Wilson SE. Keratocyte apoptosis after corneal surgery. Invest Ophthalmol Vis Sci. 1998;39(2):276-283.

19. Kim WJ, Shah S, Wilson SE. Differences in keratocyte apoptosis following transepithelial and laser-scrape photorefractive keratectomy in rabbits. J Refract Surg. 1998;14(5):526-533.

20. Chen CC, Chang J-H, Lee JB, Javier J, Azar DT. Human corneal epithelial cell viability and morphology after dilute alcohol exposure. Invest Ophthalmol Vis Sci. 2002;43(8):2593-2602.

21. Weiss RA, Liaw LH, Berns M, Amoils SP. Scanning electron microscopy comparison of corneal epithelial removal techniques before photorefractive keratectomy. J Cataract Refract Surg. 1999;25(8):1093-1096.

22. Browning AC, Shah S, Dua HS, et al. Alcohol debridement of the corneal epithelium in PRK and LASEK: an electron microscopic study. Invest Ophthalmol Vis Sci. 2003;44(2):510-513.

23. Agrawal VB, Hanuch OE, Bassage S, Aquavella JV. Alcohol versus mechanical epithelial debridement: effect on underlying cornea before excimer laser surgery. J Cataract Refract Surg. 1997;23(8):1153-1159. 


\section{Publish your work in this journal}

Clinical Ophthalmology is an international, peer-reviewed journal covering all subspecialties within ophthalmology. Key topics include: Optometry; Visual science; Pharmacology and drug therapy in eye diseases; Basic Sciences; Primary and Secondary eye care; Patient Safety and Quality of Care Improvements. This journal is indexed on
PubMed Central and CAS, and is the official journal of The Society of Clinical Ophthalmology (SCO). The manuscript management system is completely online and includes a very quick and fair peer-review system, which is all easy to use. Visit http://www.dovepress.com/ testimonials.php to read real quotes from published authors. 